\title{
Imagem corporal em adolescentes: associação com estado nutricional e atividade física
}

\author{
Body image among adolescents: the association between nutritional \\ status and physical activity
}

Edmar Roberto Fantineli (https://orcid.org/0000-0001-9536-359X) ${ }^{1}$

Michael Pereira da Silva (https://orcid.org/0000-0002-7628-3997) ${ }^{2}$

Jhonatan Gritten Campos (https://orcid.org/0000-0002-3039-2688) ${ }^{1}$

Nicolau Augusto Malta Neto (https://orcid.org/0000-0003-4385-4287) ${ }^{1}$

Ana Beatriz Pacífico (https://orcid.org/0000-0001-9719-0792) ${ }^{1}$

Wagner de Campos (http://orcid.org/0000-0003-3979-1017) ${ }^{1}$

${ }^{1}$ Setor de Ciências da Saúde, Universidade Federal do Paraná. R. Padre Camargo 280, Alto da Glória. 80060 240 Curitiba PR Brasil. edmarfantineli@

hotmail.com

${ }^{2}$ Faculdade de Medicina,

Universidade Federal do

Rio Grande. Rio Grande RS Brasil.

\begin{abstract}
The aim of this article is to verify the association between nutritional status and physical activity with the perception of body image satisfaction in a sample of adolescents from Curitiba, State of Paraná. The sample consisted of 844 adolescents of 11 to 17 years of age. The level of physical activity was evaluated by the Physical Activity Questionnaire for Adolescents. Body image perception was assessed by the Silhouette Shape Scale and the Body Shape Questionnaire. The association between nutritional status and level of physical activity with satisfaction with body image was performed through multinomial logistic regression to obtain the odds ratio with a $95 \%$ confidence interval and $p<0.05$. Overweight/obesity was positively associated with moderate and severe body image dissatisfaction (BID) among girls (OR: 6.20; 95\%CI: 2.62 - 14.66 and OR: 6.20; 95\%CI: 4.10 - 9.36) and boys (OR: 1.95; 95\%CI: 1.06 3.59 e OR: 3.91; 95\%CI: 2.57 - 5.95) and with the desire to decrease the silhouette for boys and girls (OR: 4.86; 95\%CI: 3.37 - 7.00 and OR: 6.46; 95\%CI: 4.12 - 10.14). The level of physical activity was not associated with BID. Adolescents who are overweight and obese are more likely to admit to BID. Physical activity was not related to BID.

Key words Body image, Nutritional status, Motor activity, Adolescent
\end{abstract}

Resumo Oobjetivo deste artigo é verificar a associação do estado nutricional e da atividade física com a percepção de satisfação da imagem corporal em uma amostra de adolescentes de Curitiba/PR. Compuseram a amostra 844 adolescentes entre 11 a 17 anos. O nivel de atividade física foi avaliado pelo Questionário de Atividade Física para Adolescentes. A percepção da imagem corporal foi avaliada pela Escala de Figura de Silhueta e pelo Body Shape Questionnarie. A associação do estado nutricional e do nivel de atividade física com a satisfação com a imagem corporal foi realizada através da regressão logística multinominal para obtenção da razão de chances com intervalo de confiança de $95 \%$ e $p<0,05$. O sobrepeso/obesidade esteve associado positivamente com a insatisfação com a imagem corporal (IIC) moderada e grave para meninas (RC: 6,20; IC95\%: 2,62 - 14,66 e RC: 6,20; IC95\%: 4,10 - 9,36) e meninos (RC: 1,95; IC95\%: 1,06 - 3,59 e RC: 3,91; IC95\%: 2,57 -5,95) e com o desejo de diminuir a silhueta para meninos e meninas (RC: 4,86; IC95\%: 3,37 - 7,00 e RC: 6,46, IC95\%: 4,12 - 10,14). O nivel de atividade física não se mostrou associado com a IIC. Adolescentes com sobrepeso e obesidade apresentam maior chance de reportarem IIC. A atividade física não esteve relacionada com a IIC.

Palavras-chave Imagem corporal, Estado nutricional, Atividade motora, Adolescente 


\section{Introdução}

O período da adolescência é composto por complexas transformações cognitivas, biológicas, psicológicas afetivas e sociais ${ }^{1}$. Nesta fase, surgem possíveis modificações da composição do corpo como o aumento da estatura e a massa corporal decorrentes do processo de desenvolvimento e maturação biológica². Todavia, as modificações na aparência corporal referidas ao desenvolvimento das características sexuais são muitas vezes associadas a insatisfação da imagem corporal (IIC) $)^{3-5}$.

A imagem corporal é definida como uma construção psicológica da percepção que o indivíduo tem do próprio corpo com base nas experiências adquiridas ao longo da vida ${ }^{6}$, e percepções negativas dessa imagem corporal, muitas vezes alimentadas pelo ambiente no qual o adolescente se insere, apresentam-se como um problema evidente em adolescentes do Brasil e de todo o mundo ${ }^{7}$. As constantes transformações que ocorrem no período da adolescência, como desenvolvimento puberal, torna este um período crítico para o desenvolvimento da imagem corporal ${ }^{8}$. Tal fator associa-se a outras disfunções como, transtornos alimentares, bulimia, anorexia, depressão ${ }^{9-11}$, bem como associado com variáveis neurofisiológicas e antropométricas ${ }^{12}$.

Nas últimas décadas, inúmeros esforços têm sido despendidos para compreender a percepção da imagem corporal em adolescentes onde, diversos estudos têm revelado que altos níveis de IIC são presentes em adolescentes ${ }^{7,9,13-15}$. Como exemplo, o estudo conduzido por Triches e Giugliani ${ }^{16}$, identificou uma prevalência de 63,9\% de IIC nesta população. Adicionalmente, foram encontradas diferenças entre os sexos referentes ao tamanho do corpo, onde meninas consideradas eutróficas gostariam de ter silhuetas menores e meninos de peso adequado gostariam de apresentar silhuetas maiores. Na mesma direção, Santos et al. ${ }^{17}$ identificaram que $70,9 \%$ dos adolescentes apresentaram IIC, sendo que os adolescentes com sobrepeso e baixo peso apresentaram 3,47 e 2,64 vezes mais chances, respectivamente, de relatar IIC em comparação aos eutróficos. Neste sentido, o estado nutricional pode atuar como um importante preditor da imagem corporal, tendo o sobrepeso e obesidade, como fator de risco para insatisfação com o corpo ${ }^{3,18,19}$.

Evidências da importância do estado nutricional na IIC apresentam-se mais consistentes na literatura, no entanto, dados sobre atividade física (AF) e outras variáveis que podem influen- ciar a insatisfação do adolescente, como o estágio maturacional e nível socioeconômico (NSE), apresentam-se inconsistentes na literatura ${ }^{7,20}$.

A prática regular de AF é apontada como um fator de auxílio na redução do peso corporal de indivíduos insatisfeitos com a imagem corpo$\mathrm{ral}^{21,22}$ e possibilita ainda, melhorias na autoesti$\mathrm{ma}$, na percepção e aceitação com o corpo ${ }^{23,24}$. No entanto, evidencias sobre a associação da AF com a IIC em adolescentes não se apresentam consistentes. Dados referentes a adolescentes alemães indicaram que a IIC esteve positivamente associada com o baixo nível de $\mathrm{AF}^{25}$ entretanto, outros estudos relataram não encontrar associações entre a prática de $\mathrm{AF}$ e a IIC ${ }^{17,26}$. Tais contradições denotam a necessidade de maiores investigações sobre a importância da AF na IIC e se essas associações ocorrem independentemente do estado nutricional e de demais variáveis que possam influenciar a percepção de satisfação corporal em adolescentes (faixa etária, maturação sexual e NSE). Diante disto, o objetivo deste estudo foi verificar a associação do estado nutricional e da AF com a percepção de satisfação com a imagem corporal em uma amostra de adolescentes da cidade de Curitiba, Paraná.

\section{Metodologia}

\section{Delineamento do estudo}

O presente estudo de caráter transversal utiliza dados referentes a primeira avaliação do projeto "Atividade Física e Comportamentos de Risco à Saúde em Adolescentes: Um Estudo de Coorte Prospectivo", iniciado no ano de 2015, o qual teve aprovação no Comitê de Ética em Pesquisa Envolvendo Seres Humanos (UFPR). Todos os participantes apresentaram Termo de Consentimento Livre Esclarecido assinado pelos pais/ responsáveis e também assinaram o Termo de Assentimento Livre e Esclarecido.

\section{População e amostra}

A população foi composta por adolescentes (11 a 17 anos no momento do recrutamento dos participantes), de ambos os sexos, matriculados na rede pública de ensino da cidade de Curitiba, Paraná ${ }^{27}$. A amostra foi estratificada por conglomerados respeitando a representatividade de cada Regional pertencente ao Núcleo de Educação Regional de Curitiba. Mediante sorteio aleatório dentro de cada regional de ensino, 14 esco- 
las foram selecionadas. Cada escola forneceu de 4 a 6 turmas do sexto ao primeiro ano do ensino médio que foram intencionalmente selecionadas de acordo com as possibilidades da equipe pedagógica de cada instituição de ensino.

O cálculo amostral para prevalência foi realizado adotando prevalência de 50\% de insatisfação com a imagem corporal, intervalo de confiança de $95 \%$, erro padrão de $5 \%$, efeito de delineamento de 1,5 e acréscimo de $30 \%$ para perdas e recusas. Utilizando-se do valor apresentado pelo censo escolar 2013 (135.227 alunos), a amostra mínima necessária para as estimativas de prevalência foi de 750 participantes. O presente estudo obteve dados de um total de 930 participantes, no entanto, apenas 844 apresentaram dados válidos para a realização do presente estudo resultando em uma perda de aproximadamente $10 \%$.

Realizou-se também o cálculo amostral para testes de hipóteses a posteriori através da calculadora GPower indicando que o presente número amostral foi suficiente para a obtenção de razões de chances de 1,3 com poder de 80 e alpha de $95 \%$.

\section{Procedimentos para coleta de dados}

A coleta de dados teve início no mês de agosto a novembro de 2015. As escolas sorteadas foram contatadas e informadas sobre o conteúdo e objetivos do estudo e o aceite da escola ocorreu mediante assinatura do diretor concordando com a realização dos procedimentos do estudo em sua instituição.

Os adolescentes foram convidados a participar do estudo com 2 dias de antecedência ao agendamento da coleta de dados. Estes receberam 2 cópias do termo de Consentimento Livre e Esclarecido e do Termo de Assentimento Livre e Esclarecido. A participação dos adolescentes no estudo foi concedida mediante a assinatura do TCLE por parte dos pais ou responsável legal e à assinatura do TALE por parte do adolescente.

Todos os procedimentos de coletas de dados foram realizados dentro do ambiente da escola/ colégio. Os adolescentes foram convidados a preencher questionários sobre informações sociodemográficas, atividade física, percepção de imagem corporal e foram submetidos a avaliação de peso e estatura bem com, a auto avaliação da maturação sexual. Todos os procedimentos foram realizados por uma equipe treinada de membros e colaboradores do Centro de Estudos em Atividade Física e Saúde da Universidade Federal do Paraná.

\section{Instrumentos e procedimentos}

O estado nutricional foi avaliado através do cálculo do índice de massa corporal (IMC), obtido através da relação entre a massa corporal e a estatura elevada ao quadrado. Para a classificação do estado nutricional utilizou-se das classificações dos escores $z$ de IMC para cada sexo e idade propostos pela Organização Mundial da Saúde, sendo: Baixo peso $=<-2 \mathrm{DP}$, Eutrófico $\geq$ $-2 \mathrm{DP}$ e $<+1 \mathrm{DP}$, Sobrepeso $=\geq+1 \mathrm{DP}$ e $<+2 \mathrm{DP}$ e Obesidade $=\geq+2 \mathrm{DP}^{28}$. Nenhum adolescente apresentou baixo IMC/idade, diante disso, e para fins da realização da análise, os adolescentes foram divididos em dois grupos sendo: Eutróficos e Sobrepeso/Obesidade.

O nível de AF foi avaliado utilizando-se o Questionário de Atividade Física para Adolescentes (QAFA) ${ }^{29}$. Os adolescentes que reportaram no mínimo 420 minutos de atividade física moderada a vigorosa (AFMV) semanal foram considerados como "Ativos", os demais como "Insuficientemente Ativos".

Os adolescentes foram classificados em 3 grupos de acordo com a faixa etária sendo: a) 11 a 12 anos, b) 13 a 14 anos e c) 15 anos ou mais. A maturação sexual foi avaliada através do método de auto avaliação do desenvolvimento da pilosidade pubiana com o auxílio de desenhos ${ }^{30-32}$. Para a avaliação da condição socioeconômica dos estudantes foi utilizado o questionário da Associação Brasileira de Empresas de Pesquisa ${ }^{33}$, as classificações são divididas em A1, A2, B1, B2, C1, C2, D e E, porém para a simplificação metodológica da classificação, as classes foram agrupadas em Alto (A1 e A2), Médio (B1 e B2) e Baixo (C, D e E).

Para a avaliação da imagem corporal utilizou-se o questionário autoaplicável Body Shape Questionnarie, (BSQ) desenvolvido por Cooper et al. $^{34}$ e validado para adolescentes brasileiros por Conti et al. ${ }^{35}$. O BSQ é composto de 34 questões referentes à percepção da insatisfação com aparência corporal nas últimas 4 semanas apresentando opções de respostas que variam de nunca (1) a sempre (6). O escore de insatisfação é computado pela soma dos valores das respostas podendo variar de 34 a 204. Os adolescentes foram classificados de acordo com a pontuação obtida sendo: a) Baixa insatisfação corporal $(<110$ pontos), b) Insatisfação Moderada $(\geq 110$ e $<140$ pontos) e c) insatisfação Grave ( $\geq 140$ pontos).

Adicionalmente ao BSQ, utilizou-se a Escala de Silhueta de Thompson e Gray ${ }^{36}$ validada para adolescentes brasileiros por Conti e Latorre ${ }^{14}$ como mais um instrumento de identificação de 
insatisfação com a aparência corporal. Esta escala é composta por duas questões referentes a sua aparência atual (Escore de silhueta atual) e sua aparência desejada (Escore de silhueta ideal). Para cada questão 9 silhuetas (de ambos os sexos) foram apresentadas representando condições de magreza extrema à níveis de obesidade. Os adolescentes foram orientados a assinalarem, primeiro, qual silhueta melhor representava sua aparência atual e, segundo qual silhueta melhor representava uma aparência desejada (ideal). A insatisfação corporal foi obtida através do resultado do seguinte cálculo: Escore da silhueta atual - Escore da silhueta ideal. Os adolescentes que apresentam valores positivos neste cálculo foram classificados na categoria "Deseja diminuir" e os que apresentam valores negativos, foram classificados na categoria "Deseja aumentar" e aqueles que apresentam valores igual a zero estavam "Satisfeitos".

A utilização desses dois instrumentos traz informações sobre a percepção da imagem corporal de duas formas diferentes, mas não divergentes. O BSQ apresenta uma análise mais ampla de indicadores comportamentais e psicológicos associados a IIC favorecendo uma avaliação mais global deste componente ${ }^{18,34}$. Já a escala de silhueta fornece informações específicas sobre fatores estéticos que denotam insatisfação com as formas corporais apresentadas pelo adolescente ${ }^{36,37}$.

\section{Análise estatística}

A estatística descritiva foi utilizada para a caracterização dos participantes utilizando medidas de tendência central e dispersão, bem como distribuição de frequência das variáveis categóricas. Todas as análises foram realizadas separadamente para cada sexo. O teste de Qui-Quadrado foi utilizado para verificar possíveis diferenças entre os sexos para variáveis categóricas.

Para verificar a associação do estado nutricional, do nível de AF, faixa etária, maturação sexual e NSE com a percepção de satisfação com a imagem corporal dos adolescentes foi utilizada a regressão logística multinominal para obtenção de razão de chances (RC) com intervalos de confiança de 95\% (IC95\%). A Tabela 1 apresenta o quadro de operacionalização de variáveis e sua presença em cada modelo de análise. Devido as características de amostragem complexa, pesos amostrais e ajuste robusto de erros por conglomerado (Escolas) foram aplicados. Para todas as análises adotou-se $\mathrm{p}<0,05$ como significância estatística. Todas as análises foram realizadas utilizando o software Stata MP 13.0.

\section{Resultados}

A amostra do presente estudo foi composta por 844 adolescentes, sendo 401 (47,51\%) meninos e 443 (52,49\%) meninas com idade média de 13,8 $( \pm 1,76)$ anos. Em relação a prática de AFMV, verificou-se que os adolescentes atingiram uma média total de $146,1 \pm 176,1$ minutos por semana, sendo a média dos meninos de 179,8 \pm 185,5 e das meninas $113,3 \pm 160,1$. Em relação ao IMC verificou-se valores médios $21,1 \pm 4,0$ para meninos e 21,6 $\pm 4,2$ para meninas. A amostra foi composta em sua maioria por adolescentes na faixa etária entre 15 anos ou mais de idade $(35,54 \%)$, pertencentes a classe social B (56,75\%), e com o estado nutricional eutrófico (60,31\%). Diferenças significativas entre os sexos ocorreram na Insatisfação grave (Meninos: 5,24\%; Meninas: 17,83\%, p < 0,01), desejo de Diminuir a Silhueta (Meninos: 43,14\%; Meninas: 59,59\%, p < 0,01), Desejo de Aumentar a Silhueta (Meninos: 28,18\%; Meninas: 18,06\%, p < 0,01) e praticar ao menos 420 minutos por semana de AFMV (Meninos: 72,32\%; Meninas: 50,11\%, p < 0,01). Estes resultados são apresentados na Tabela 2.

Em relação aos fatores associados a IIC obtida através do questionário BSQ (Tabela 3), verificou-se que o estado nutricional esteve positivamente relacionado com a insatisfação moderada para ambos os sexos. Meninos com sobrepeso/ obesidade apresentaram 1,95 vezes mais chances (IC95\%: 1,06 - 3,59) de estarem no grupo que indicou um grau moderado de insatisfação, já as meninas com sobrepeso/obesidade, apresentaram 3,95 (IC95\%: 2,57 - 5,95) mais chances de também estarem no grupo de insatisfação moderada.

Para a insatisfação grave, verificou-se que, para o sexo feminino as variáveis de faixa etária, maturação sexual, NSE e estado nutricional estiveram positivamente associadas a esta variável sendo que, as meninas com 13 a 14 anos (RC: 4,72; IC95\%: 2,14 - 10,40), e as de 15 anos ou mais (RC: 7,09; IC95\%: 2,75 - 18,26), nos maiores estágios maturacionais (RC: 1,32; IC95\%: 1,05 - 1,64), de alto NSE e que apresentavam sobrepeso/obesidade (RC: 1,87; IC95\%: 1,10 - 3,22) apresentaram mais chances de estarem no grupo de insatisfação grave. Para os meninos apenas o estado nutricional esteve positivamente associado com a insatisfação grave (RC: 3,91; IC95\%: 2,57 - 5,95). O nível de AF não esteve associado com nenhuma das variáveis de IIC avaliados através do questionário BSQ. 
Tabela 1. Operacionalização e inserção de variáveis nos modelos de regressão multinomiais para a associação com a insatisfação com a imagem corporal.

\begin{tabular}{|c|c|c|c|}
\hline \multirow{3}{*}{$\begin{array}{c}\text { Variáveis } \\
\text { Independentes }\end{array}$} & \multirow{3}{*}{ Operacionalização } & \multicolumn{2}{|c|}{ Variáveis dependentes } \\
\hline & & Insatisfação BSQ & $\begin{array}{c}\text { Insatisfação Escala de } \\
\text { Silhueta }\end{array}$ \\
\hline & & $\begin{array}{c}0=\text { Satisfeito } \\
1=\text { Insatisfação Moderada } \\
2=\text { Insatisfação Grave }\end{array}$ & $\begin{array}{c}0=\text { Satisfeito } \\
1=\text { Deseja reduzir } \\
2=\text { Deseja aumentar }\end{array}$ \\
\hline Faixa Etária & $\begin{array}{l}0=11 \text { a } 12 \text { Anos } \\
1=13 \text { a } 14 \text { Anos } \\
2=15 \text { Anos ou mais }\end{array}$ & + & + \\
\hline Maturação Sexual & 1 a 5 & + & + \\
\hline NSE & $\begin{array}{l}0=\text { Baixo }(\text { Classes } C, D \text { e E) } \\
1=\text { Médio }(B 1 \text { e B2) } \\
2=\text { Alto }(A)\end{array}$ & + & + \\
\hline $\begin{array}{l}\text { Estado } \\
\text { Nutricional }\end{array}$ & $\begin{array}{l}0=\text { Peso Normal } \\
1=\text { Sobrepeso } / \text { Obesidade }\end{array}$ & + & + \\
\hline Atividade Física & $\begin{array}{l}0=\mathrm{AFMV} \geq 420 \mathrm{~min} / \mathrm{semana} \\
1=\mathrm{AFMV}<420 \mathrm{~min} / \mathrm{semana}\end{array}$ & + & + \\
\hline
\end{tabular}

+: indicam entrada nos modelos de regressão. BSQ: Body shape questionnaire. NSE: Nível socioeconômico. AFMV: Atividade física de moderada a vigorosa intensidade.

Em relação aos fatores associados a insatisfação da imagem corporal avaliados através da escala de Silhueta corporal (Tabela 4) verificouse que, para o sexo masculino, somente o estado nutricional (RC: 4,86; IC95\%: 3,37 - 7,00) esteve positivamente associado com o desejo de diminuir a silhueta. Já para o sexo feminino, as variáveis de maturação sexual, e estado nutricional estiveram positivamente associadas com o desejo de diminuir a silhueta corporal, onde as meninas com estágios de maturação avançados (RC: 1,43; IC95\%: 1,04 - 1,95), e com sobrepeso/obesidade (RC: 6,46; IC95\%: 4,12 - 10,14) apresentam mais chances quando comparadas a categoria de referência. Adicionalmente, o alto NSE mostrou-se inversamente associado ao desejo de diminuir a silhueta corporal em relação a categoria de referência (RC: 0,45; IC95\%: 0,22 - 0,88).

Com relação ao desejo de aumentar a silhueta, o estado nutricional mostrou-se inversamente associado a esta variável para ambos os sexos. Meninos com sobrepeso/obesidade apresentaram 88\% (RC: 0,12; IC95\%: 0,04 - 0,31) e meninas 76\% menos chances de reportarem o desejo de aumentar a silhueta corporal em relação a categoria de referência. Adicionalmente, para o sexo feminino, o NSE Médio: (RC: 0,36; IC95\%: 0,13 - 0,99) e Alto: (RC: 0,37; IC95\%: 0,15 - 0,93) também apresentaram associação inversa com o desejo de aumentar as silhuetas. Adicionalmen- te, a maturação sexual associou-se positivamente com o desejo de aumentar a silhueta no sexo feminino. Meninas com estágios de maturação avançados apresentaram 1,38 mais chances (IC95\%: 1,02 - 1,87) de estarem no grupo que apresentou desejo de aumentar a silhueta. A AF não se associou a nenhuma das condições de insatisfação avaliadas pela escala de silhuetas.

\section{Discussão}

O presente estudo teve como objetivo verificar a associação do estado nutricional e da AF com a percepção da imagem corporal em uma amostra de adolescentes matriculados em escolas públicas da cidade de Curitiba-PR mediante utilização de dois instrumentos previamente validados para a utilização em adolescentes brasileiros (BSQ e Escala de Silhueta $)^{14,35}$. A adolescência apresenta-se como um período de grandes transformações e caracterizado pela adoção de diversos comportamentos que podem favorecer ou não a saúde. E nessa fase da vida, a forma com que esses adolescentes percebem seu corpo parece influenciar alguns comportamentos e condições patológicas de grande impacto na vida dos jovens como por exemplo, transtornos alimentares (bulimia, e anorexia) e depressão $o^{9-11}$. O presente estudo identificou claramente que alterações do peso 


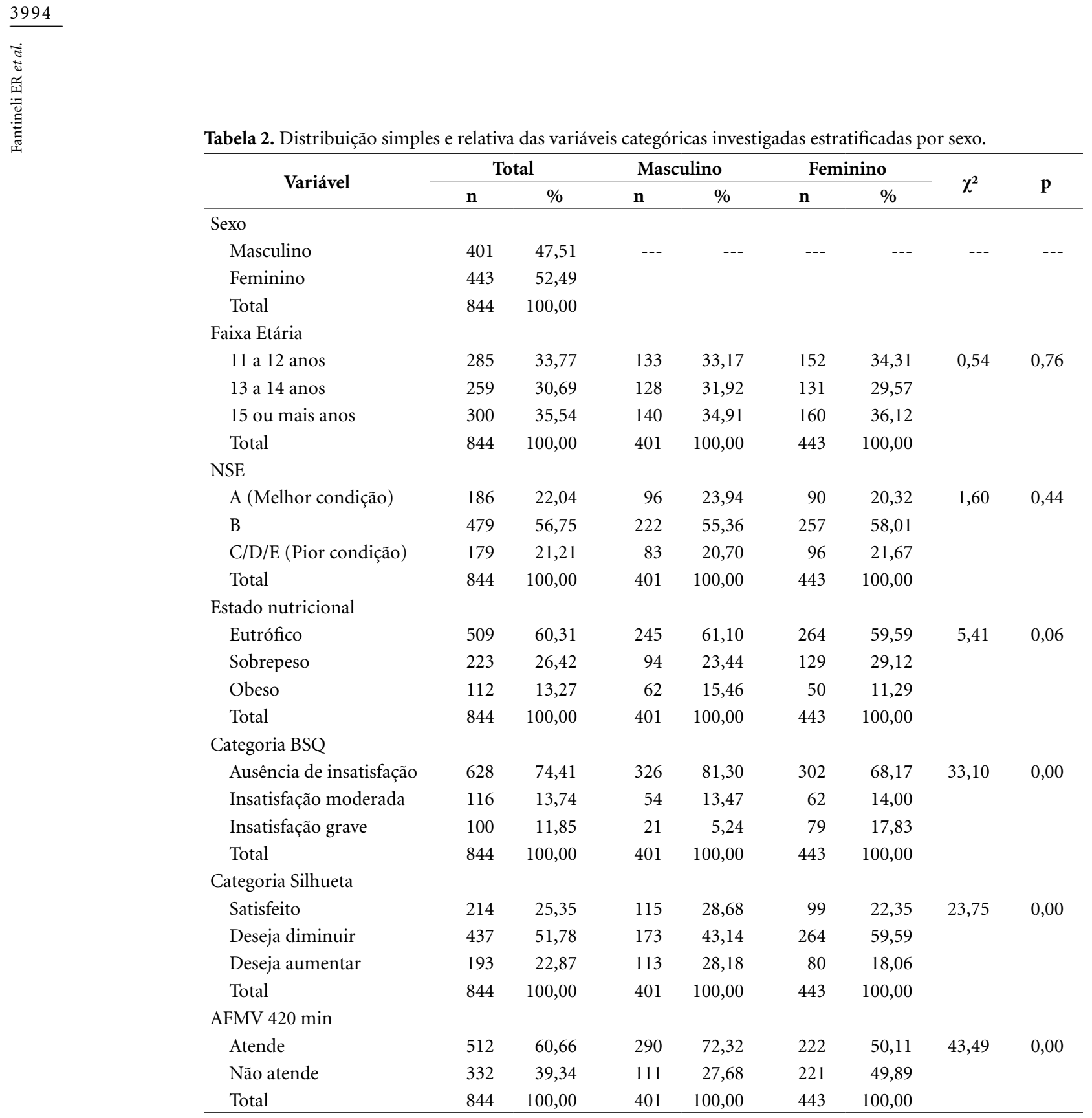

corporal (sobrepeso/obesidade) são importantes preditores de insatisfação com a imagem corporal tanto em meninos quanto em meninas e tal associação ocorre independentemente da faixa etária, maturação biológica, NSE e nível de atividade física. Esta por sua vez, parece não influenciar tal percepção na amostra de adolescentes participantes desse estudo.

Em relação aos dados descritivos apresentados pelo BSQ, verificou-se que $25,59 \%$ dos adolescentes apresentaram algum grau de IIC, sendo a insatisfação grave mais presente nas meninas quando comparada aos meninos $(17,83 \%$ vs. $\left.5,24 \%, \chi^{2}=33,10, p=0,00\right)$. Os resultados cor- roboram com o estudo de Laus et al. ${ }^{18}$ em que, $14 \%$ dos meninos e $60 \%$ das meninas apresentaram algum grau de distorção da imagem, onde a distorção mais intensa foi mais prevalente para meninas (25\%). Em relação a análise de associação, o presente estudo mostrou que adolescentes de ambos os sexos com sobrepeso/obesidade apresentaram maior probabilidade de também reportarem a insatisfação corporal moderada e grave. $\mathrm{O}$ estudo de Martins et al. ${ }^{10}$, avaliando a IIC através do BSQ, também identificou as alterações de peso corporal como importante fator associado a IIC. Neste estudo, adolescentes do sexo feminino com excesso de peso apresentaram 
Tabela 3. Razão de chances (RC) e intervalo de confiança de 95\% (IC 95\%) para os fatores associados a insatisfação moderada e grave obtida pelo BSQ.

\begin{tabular}{|c|c|c|c|c|c|c|c|c|}
\hline \multirow{3}{*}{ Variável } & \multicolumn{4}{|c|}{ Insatisfação Moderada } & \multicolumn{4}{|c|}{ Insatisfação Grave } \\
\hline & \multicolumn{2}{|l|}{ Masculino } & \multicolumn{2}{|l|}{ Feminino } & \multicolumn{2}{|l|}{ Masculino } & \multicolumn{2}{|l|}{ Feminino } \\
\hline & $\begin{array}{c}\text { RC } \\
\text { (IC 95\%) }\end{array}$ & $\mathbf{p}$ & $\begin{array}{c}\text { RC } \\
\text { (IC 95\%) }\end{array}$ & $\mathbf{p}$ & $\begin{array}{c}\text { RC } \\
\text { (IC 95\%) }\end{array}$ & $\mathbf{p}$ & $\begin{array}{c}\text { RC } \\
\text { (IC 95\%) }\end{array}$ & $\mathbf{p}$ \\
\hline \multicolumn{9}{|l|}{ Faixa Etária } \\
\hline 11 a 12 Anos & \multicolumn{2}{|l|}{1} & \multicolumn{2}{|l|}{1} & \multicolumn{2}{|l|}{1} & \multicolumn{2}{|c|}{1} \\
\hline 13 a 14 Anos & $0,36(0,13-1,01)$ & 0,05 & $0,91(0,38-2,17)$ & 0,84 & $0,90(0,17-4,81)$ & 0,90 & $4,72(2,14-10,40)$ & 0,00 \\
\hline 15 Anos ou mais & $0,42(0,09-1,80)$ & 0,24 & $1,74(0,55-5,52)$ & 0,35 & $1,40(0,31-6,16)$ & 0,65 & $7,09(2,75-18,26)$ & 0,00 \\
\hline Maturação sexual & $1,26(0,75-2,10)$ & 0,37 & $1,15(0,65-2,03)$ & 0,62 & $1,01(0,69-1,47)$ & 0,96 & $1,32(1,05-1,64)$ & 0,01 \\
\hline \multicolumn{9}{|l|}{ NSE } \\
\hline Baixo (C/D/E) & \multicolumn{2}{|l|}{1} & \multicolumn{2}{|l|}{1} & \multicolumn{2}{|l|}{1} & \multicolumn{2}{|c|}{1} \\
\hline Médio (B) & $1,11(0,48-2,56)$ & 0,80 & $0,60(0,33-1,10)$ & 0,10 & $1,63(0,40-6,55)$ & 0,49 & $1,41(0,53-3,74)$ & 0,49 \\
\hline Alto (A) & $1,06(0,38-2,97)$ & 0,91 & $0,70(0,23-2,11)$ & 0,53 & $4,35(0,80-19,62)$ & 0,91 & $1,87(1,10-3,22)$ & 0,02 \\
\hline \multicolumn{9}{|l|}{ Estado Nutricional } \\
\hline Eutrófico & \multicolumn{2}{|l|}{1} & \multicolumn{2}{|l|}{1} & \multicolumn{2}{|l|}{1} & \multicolumn{2}{|l|}{1} \\
\hline $\begin{array}{l}\text { Sobrepeso/ } \\
\text { Obesidade }\end{array}$ & $1,95(1,06-3,59)$ & 0,03 & $3,91(2,57-5,95)$ & 0,00 & $6,20(2,62-14,66)$ & 0,00 & $6,20(4,10-9,36)$ & 0,00 \\
\hline \multicolumn{9}{|l|}{ Atividade Física } \\
\hline $\begin{array}{l}\text { AFMV } \geq 420 \\
\mathrm{~min} / \mathrm{semana}\end{array}$ & \multicolumn{2}{|l|}{1} & \multicolumn{2}{|l|}{1} & \multicolumn{2}{|l|}{1} & \multicolumn{2}{|l|}{1} \\
\hline $\begin{array}{l}\text { AFMV }<420 \\
\mathrm{~min} / \text { semana }\end{array}$ & $1,48(0,49-4,42)$ & 0,44 & $0,59(0,31-1,12)$ & 0,11 & $0,75(0,24-2,16)$ & 0,60 & $0,90(0,65-1,23)$ & 0,52 \\
\hline
\end{tabular}

Tabela 4. Razão de chances (RC) e intervalo de confiança de 95\% (IC 95\%) para os fatores associados ao desejo de aumentar ou diminuir a silhueta.

\begin{tabular}{|c|c|c|c|c|c|c|c|c|}
\hline \multirow{4}{*}{ Variável } & \multicolumn{8}{|c|}{ Insatisfação Silhueta } \\
\hline & \multicolumn{2}{|l|}{ Masculino } & \multicolumn{2}{|l|}{ Feminino } & \multicolumn{2}{|l|}{ Masculino } & \multicolumn{2}{|l|}{ Feminino } \\
\hline & \multicolumn{4}{|c|}{ Deseja Diminuir } & \multicolumn{4}{|c|}{ Deseja Aumentar } \\
\hline & $\begin{array}{c}\text { RC } \\
(\text { IC 95\%) }\end{array}$ & $\mathbf{p}$ & $\begin{array}{c}\text { RC } \\
(\text { IC } 95 \%)\end{array}$ & $\mathbf{p}$ & $\begin{array}{c}\text { RC } \\
(\text { IC 95\%) }\end{array}$ & $\mathbf{p}$ & $\begin{array}{c}\text { RC } \\
(\text { IC } 95 \%)\end{array}$ & $\mathbf{p}$ \\
\hline \multicolumn{9}{|l|}{ Faixa Etária } \\
\hline 11 A 12 Anos & \multicolumn{2}{|l|}{1} & \multicolumn{2}{|l|}{1} & \multicolumn{2}{|l|}{1} & \multicolumn{2}{|l|}{1} \\
\hline 13 A 14 Anos & $0.96(0,52-1,79)$ & 0,92 & $1,09(0,48-2,53)$ & 0,82 & $0,84(0,27-2,58)$ & 0,76 & $0,75(0,34-1,64)$ & 0,47 \\
\hline 15 Anos ou mais & $0,88(0,47-1,63)$ & 0,69 & $1,16(0,44-3,07)$ & 0,76 & $1,24(, 36-4,26)$ & 0,73 & $0,73(0,27-1,95)$ & 0,53 \\
\hline Maturação sexual & $0,85(0,59-1,22)$ & 0,39 & $1,43(1,04-1,95)$ & 0,02 & $0,96(0,51-1,74)$ & 0,86 & $1,38(1,02-1,87)$ & 0,03 \\
\hline \multicolumn{9}{|l|}{ NSE } \\
\hline Baixo (C/D/E) & \multicolumn{2}{|l|}{1} & \multicolumn{2}{|l|}{1} & \multicolumn{2}{|l|}{1} & \multicolumn{2}{|l|}{1} \\
\hline Médio (B) & $0,70(0,30-1,59)$ & 0,39 & $0,42(0,18-1,05)$ & 0,06 & $1,81(0,73-4,47)$ & 0,19 & $0,36(0,13-0,99)$ & 0,04 \\
\hline Alto (A) & $0,81(0,24-2,70)$ & 0,74 & $0,45(0,22-0,88)$ & 0,02 & $1,48(0,49-4,45)$ & 0,48 & $0,37(0,15-0,93)$ & 0,03 \\
\hline \multicolumn{9}{|l|}{ Estado Nutricional } \\
\hline Eutrófico & \multicolumn{2}{|l|}{1} & \multicolumn{2}{|l|}{1} & \multicolumn{2}{|l|}{1} & \multicolumn{2}{|l|}{1} \\
\hline Sobrepeso/Obesidade & $4,86(3,37-7,00)$ & 0,00 & $6,46(4,12-10,14)$ & 0,00 & $0,12(0,04-0,31)$ & 0,00 & $0,24(0,08-0,71)$ & 0,01 \\
\hline \multicolumn{9}{|l|}{ Atividade Física } \\
\hline $\begin{array}{l}\mathrm{AFMV} \geq 420 \mathrm{~min} / \\
\text { semana }\end{array}$ & \multicolumn{2}{|l|}{1} & \multicolumn{2}{|l|}{1} & \multicolumn{2}{|l|}{1} & \multicolumn{2}{|l|}{1} \\
\hline $\begin{array}{l}\text { AFMV }<420 \mathrm{~min} / \\
\text { semana }\end{array}$ & $1,50(0,84-2,66)$ & 0,17 & $1,00(0,56-1,78)$ & & $1,47(0,73-2,98)$ & 0,28 & $0,98(0,45-2,12)$ & 0,95 \\
\hline
\end{tabular}


2,64 vezes mais chance de terem IIC em relação àquelas com peso normal. No entanto, os autores optaram pela classificação da IIC de forma dicotômica, ou seja, presença ou ausência de insatisfação, impossibilitando a comparação direta com o presente estudo que apresenta dois níveis referentes a presença de IIC (leve e grave). Na mesma direção, Petroski et al. ${ }^{15}$ encontraram que os adolescentes com excesso de peso, tiveram 8,45 vezes mais chance de apresentarem IIC quando comparados com indivíduos eutróficos.

Contudo, é importante ressaltar que a IIC não é característica somente dos adolescentes com sobrepeso e obesidade. Estudos apontam que os adolescentes eutróficos ou classificados abaixo do peso, também apresentam IIC, como o reportado por Paludo e Dalpube ${ }^{38}$, que indicaram que dentre os indivíduos classificados como eutróficos, $34,6 \%$ deles mostraram-se insatisfeitos com sua imagem corporal e, dentre os adolescentes classificados com estado nutricional abaixo do peso, $21,2 \%$ desejavam ser ainda mais magros.

A AF não se apresentou associada a nenhum dos indicadores de IIC, tanto para meninos quanto para meninas em nosso estudo, resultados que divergem do apresentado por com Finne et al. ${ }^{25}$, onde foi apontado que a IIC esteve associada a inatividade física entre os adolescentes. Ainda, Daniel et al.$^{39}$ apontam que a maior proporção dos adolescentes que apresentaram IIC esteve associado a uma menor frequência e um menor tempo de prática de AF na semana. Porém, não está claro se os baixos níveis de AF determinam a IIC ou se uma imagem corporal negativa leva a menores níveis de $\mathrm{AF}$ e se essa associação pode ser mediada pelo estado nutricional.

Contudo, independente da inconsistência da literatura, baixos níveis de AF em adolescentes brasileiros vem sendo reportados em estudos nacionais apresentando-se como possível agravante para a IIC visto a sua associação com o aparecimento de sobrepreso e obesidade. Dados da Pesquisa Nacional de Saúde do Escolar (PENSE $)^{40}$ de 2015 verificaram que apenas $34,4 \%$ dos adolescentes indicaram atender o mínimo de 300 minutos de AFMV por semana. No presente estudo, verificou-se altas prevalências de prática de AFMV tanto em meninos quanto em meninas quando comparado ao relatado pelo PENSE. Fator esse que pode ser atribuído a diferenças no instrumento de medida utilizado. Podendo o instrumento do presente estudo ter superestimado os minutos em AFMV nos adolescentes.

Para os dados apresentados pela escala de silhueta, verificou-se que $74,65 \%$ dos adoles- centes indicaram algum nível de IIC (Desejo de Aumentar + Desejo de Diminuir). Os meninos apresentaram maior proporção em desejar aumentar a silhueta (Meninos: $28,18 \%$ vs. Meninas: $18,06 \%$ ) e o inverso foi visto em relação ao desejo de diminuir a silhueta (Meninos: $43,14 \%$ vs. Meninas: $59,59 \%, 2=23,75, p<0,01)$. A prevalência verificada no presente estudo apresenta-se superior à de alguns estudos nacionais. Fidelix et al. ${ }^{41}$, verificaram IIC em $56,5 \%$ dos adolescentes com idades entre 14 a 17 anos em uma cidade de pequeno porte, no estado de Minas Gerais. Resultados semelhantes (59\%), foram encontrados por Vilela et al. ${ }^{42}$ em escolares de 7 a 19 anos em Porto Alegre, RS. Em relação a associação do estado nutricional com a IIC avaliada pela escala de silhueta, como esperado, tanto meninos quanto meninas com sobrepeso e obesidade apresentaram maiores chances de desejarem diminuir a silhueta. Tais resultados são reportados pelos achados de Dumith et al. ${ }^{7}$ sobre a IIC em adolescentes, os quais apontam que dentre os classificados como "obesos", mais de $90 \%$ desejavam diminuir a silhueta.

Assim como no BSQ, a AF não se apresentou associada a IIC através da escala de silhueta corporal entre os adolescentes. Na mesma direção, Adami et al. ${ }^{43}$ não encontraram associação entre a satisfação com a silhueta corporal e o nível de AF bem como em, estudo conduzido por Mota el al. ${ }^{44}$ onde não foram encontradas associações da imagem corporal dos adolescentes e a prática de AF dos mesmos. Uma possível explicação para a não associação destas variáveis é de que os adolescentes podem não considerar a $\mathrm{AF}$ como uma ferramenta para a melhora da sua percepção da imagem corporal, assim como as opções de atividades físicas oferecidas pelas escolas não serem favoráveis para que haja associações significativas e possível influência na percepção da imagem corporal desta população.

Estes resultados de associações visualizados em ambos os instrumentos utilizados para a avaliação da IIC em adolescentes reforçam que o sobrepeso e a obesidade apresentam-se como um importante favorecedor de uma maior insatisfação entre os adolescentes. A exposição à "cultura do magro", imposto pelos meios de comunicação, apresentam grande influencia nessa percepção visto que oferta um padrão ideal de beleza que na grande maioria das vezes é inatingível, principalmente em indivíduos com sobrepeso e obesidade $^{45}$. Esta magreza ideal nem sempre atingível desencadeia um aumento na predisposição à IIC que se manifesta, de acordo com Alves et al. ${ }^{46}$, 
tanto nas meninas quanto nos meninos como resultado direto do não enquadramento nos padrões estéticos culturais da sociedade. Silva et al. ${ }^{47}$ citam que esses padrões estéticos culturais da sociedade, envolvem não somente ter um corpo magro e sim uma construção de ser saudável, se integrando a multidimensões da vida humana, relacionadas por determinantes de ordem político-social, cultural e econômica, evidenciadas por necessidades de saúde, o que engloba uma alimentação saudável, prática de AF, cuidados com a saúde, dentre outros. Segundo os autores, no que se refere a alimentação, os adolescentes possuem boa apreensão sobre o que é ser saudável, embora muitas vezes não coloquem isso em prática.

Alves et al. ${ }^{46}$ indicam também que, atualmente, estamos imersos em uma época que favorece divinização do corpo belo. Tal característica por sua vez, é proveniente de uma construção cultural que imputa informações que definem padrões ideais de beleza que são divulgados constantemente através de diversos meios de comunicação e interação social. Locais, como academias de ginásticas, consultórios e centros de tratamento estéticos atuam, segundo Sampaio e Ferreira ${ }^{48}$ como fábricas produtoras desse padrão de corpo ideal podendo expor, não só os indivíduos com sobrepeso e obesidade, mas também indivíduos sem alterações de peso corporal a padrões corporais inatingíveis. De fato, tais padrões corporais como produto de imputações culturais e de relações sociais, está em constante modificação e, no adolescente, sendo esse um grande consumidor vulnerável à influências sociais, estes padrões podem causar grande impacto no desenvolvimento psicossocial do mesmo, influenciando, como abordado anteriormente, a maiores níveis de IIC.

Associações importantes também foram verificadas para variáveis de cunho biológico e sócio demográficos no sexo feminino. A maturação sexual foi um importante preditor da insatisfação corporal grave nas meninas, onde com avanço nos estágios maturacionais elas apresentaram mais chances de apresentarem insatisfação corporal grave. O mesmo padrão de associação foi visto para a faixa etária, onde meninas mais velhas (13 a 14 anos e 15 anos ou mais) tiveram mais chances de apresentarem insatisfação corporal quando comparadas as mais jovens (11 e 12 anos). No mesmo sentido, Scherer et al. ${ }^{20}$ ao verificar a associação da imagem corporal com a maturação sexual em adolescentes do sexo feminino, apontam que dentre as adolescentes que gostariam de aumentar o peso corporal, a maior proporção estava ainda no processo de maturação sexual. Por outro lado, naquelas que gostariam de reduzir o peso corporal, a maioria delas já estavam no estágio final de maturação.

Com relação ao NSE, o presente estudo mostrou que as meninas com alto NSE tinham mais chance de apresentarem insatisfação corporal grave. De forma contrária ao visualizado no presente estudo, Dumith et al. ${ }^{7}$ apresentou relações entre a IIC e a situação econômica indicando que as meninas com maior nível socioeconômico são mais satisfeitas com a imagem corporal quando comparado com as classes mais baixas.

Reforçando argumentação apresentada anteriormente sobre o impacto psicossocial de IIC em adolescentes, ressalta-se achados recentes de fatores associados à IIC em adolescentes brasileiros que, além das associações claras vistas para o sexo feminino e para o sobrepeso/obesidade, as quais corroboram com o presente achado, os autores identificaram que fatores como consumo de álcool, sentimentos de tristeza e pensamentos suicidas estiveram positivamente associados à $\mathrm{IIC}^{49}$. Tais resultados agregam ao impacto negativo que estas alterações da percepção da imagem corporal causam em comportamentos e fatores de risco psicossociais em adolescentes.

O presente estudo não é livre de limitações. Denota-se que o instrumento para medida de AF pode favorecer a superestimação desta variável. O baixo número de adolescentes classificados com obesidade limitou uma análise mais detalhada sobre as associações do estado nutricional com a IIC. No entanto, as associações encontradas convergem para o que é apresentado na literatura. Outra possível limitação, seria a não utilização de dados referentes ao consumo alimentar dos adolescentes, visto que o mesmo é também um determinante para a composição corporal, estando diretamente relacionado a IIC. Ainda, cabe ressaltar que a amostra coletada não pôde representar os adolescentes matriculados no ensino público estadual da cidade de Curitiba-PR, no entanto faz-se necessário reforçar as qualidades apresentadas neste estudo em relação a seleção amostral, tais como, a seleção aleatória de escolas dentro de cada distrito educacional do município a qual favoreceu uma maior variabilidade amostral.

\section{Conclusão}

Conclui-se que o estado nutricional foi um importante indicador para a IIC, sendo que os ado- 
lescentes com sobrepeso e obesidade apresentam maior chance de reportarem IIC. A AF não esteve relacionada com a IIC, independente do instrumento utilizado. Adicionalmente, meninas mais velhas, em estágios maturacionais mais elevados e com alto NSE apresentaram mais chances de apresentarem IIC.

Os dados da presente investigação fornecem, apesar das limitações, informações importantes sobre fatores associados a insatisfação com a imagem corporal em adolescentes. O sobrepeso e obesidade para ambos os sexos, o estágio maturacional elevado e o alto NSE em meninas indicam condições ou estratos mais propensos a essa condição. Sabe-se que a IIC possui relações importantes com transtornos alimentares como anorexia e bulimia e também com fatores psicopatológicos como depressão. Diante disso, entender quais grupos apresentam-se mais susceptíveis a essa condição pode auxiliar a proposição de intervenções direcionadas, favorecendo a adoção de comportamentos saudáveis como hábitos alimentares e a prática de AF. O âmbito escolar pode auxiliar na orientação dos estudantes à adoção desses comportamentos, além da relação do adolescente com o próprio corpo, tendo em vista que nesta fase da vida ocorrem diversas mudanças físicas, psicológicas, cognitivas e sociais. Por conta disso, políticas públicas voltadas ao meio escolar se tornam necessárias, visando estratégias e planejamentos para uma maior aceitação do adolescente com o seu corpo.

\section{Colaboradores}

ER Fantineli: delineamento, análise, interpretação dos dados e redação do artigo. MP Silva: delineamento, análise, interpretação dos dados e redação do artigo. JG Campos: redação do artigo e revisão crítica. NA Malta Neto: redação do artigo e revisão crítica. $\mathrm{AB}$ Pacífico: redação do artigo e revisão crítica. W Campos: revisão crítica e aprovação da versão a ser publicada. 


\section{Referências}

1. World Health Organization (WHO). Global recommendations on physical activity for health. Genebra: WHO; 2010.

2. Malina RM, Bouchard C, Bar-Or O. Growth, Maturation, and Physical Activity. Champaign: Human Kinetics; 2004.

3. Paxton SJ, Eisenberg ME, Neumark-Sztainer D. Prospective predictors of body dissatisfaction in adolescent girls and boys: a five-year longitudinal study. Dev Psychol 2006 ;42(5):888-899.

4. Petroski EL, Velho NM, Bem MFL. Idade de menarca e satisfação com o peso corporal. Rev Bras Cineantropometria e Desempenho Hum 1999; 1(1):30-36.

5. Conti MA, Gambardella AMD, Frutuoso MFP. Insatisfação com a imagem corporal em adolescentes e sua relação com a maturação sexual. Rev Bras Cresc Desenvolv Hum 2005; 15(2):36-44.

6. Cash TF, Szymanski ML. The Development and Validation of the Body-Image Ideals Questionnaire. J Pers Assess 1995; 64(3):466-477.

7. Dumith SC, Menezes AMB, Bielemann RM, Petresco S, Silva ICM, Linhares RS, Amorim TC, Duarte DV, Araújo CLP, Santos JV. Insatisfação corporal em adolescentes: um estudo de base populacional. Cien Saude Colet 2012; 17(9):2499-2505.

8. Braga PD, Molina CB, Martins A. Representações do corpo : com a palavra um grupo de adolescentes de classes populares. Cien Saude Colet 2010; 15(1):87-95.

9. Fortes LS, Ferreira EMC. Comparação da insatisfação corporal e do comportamento alimentar inadequado em atletas adolescentes de diferentes modalidades esportivas. Rev Bras Educ Física Esporte 2011; 25(4):707716.

10. Martins CR, Pelegrini A, Matheus SC, Petroski EL. Insatisfação com a imagem corporal e relação com estado nutricional, adiposidade corporal e sintomas de anorexia e bulimia em adolescentes. Rev Psiquiatr RS 2010; 32(1):19-23.

11. Stice E, Bearman SK. Body-image and eating disturbances prospectively predict increases in depressive symptoms in adolescent girls: a growth curve analysis. Dev Psychol 2001; 37(5):597-607.

12. Felden ÉPG, Claumann GS, Sacomori C, Daronco LSE, Cardoso FL, Pelegrini A. Fatores sociodemográficos e imagem corporal em adolescentes do ensino médio. Cien Saude Colet 2015; 20(11):3329-3338.

13. Malete L, Motlhoiwa K, Shaibu S, Wrotniak BH, Maruapula SD, Jackson J, Compher CW. Body image dissatisfaction is increased in male and overweight/obese adolescents in Botswana. J Obes 2013; 2013:763624.

14. Conti MA, Latorre MRDO. Estudo de validação e reprodutibilidade de uma escala de silhueta para adolescentes. Psicol Estud 2009; 14(4):699-706.

15. Petroski EL, Pelegrini A, Glaner MF. Insatisfação corporal em adolescentes rurais e urbanos. Motricidade 2009; 5(4):13-25.

16. Triches RM, Giugliani ERJ. Insatisfação corporal em escolares de dois municípios da região Sul do Brasil. Rev Nutr 2007; 20(2):119-128.

17. Santos JFS, Oliveira P, Campos AA, Pereira EO, Sousa EA. Relação entre Insatisfação Corporal e Atividade Física em Adolescentes da Zona Urbana de Irati-PR. Rev da Educ Física/UEM 2014; 25(2):193.
18. Laus MF, Costa TMB, Almeida SS. Body image dissatisfaction and its relationship with physical activity and body mass index in Brazilian adolescents. J Bras Psiquiatr 2011; 60(4):315-320.

19. Battisti M, Bergjohann P, Adami FS, Fassina P. Percepção da imagem corporal associada ao estado nutricional de crianças e adolescentes. Rev Bras Promoção Saúde 2017; 30(1):86-92.

20. Scherer FC, Martins CR, Pelegrini A, Petroski EL. Imagem corporal em adolescentes : associação com a maturação sexual e sintomas de transtornos alimentares. J Bras Psiquiatr 2010; 59(3):198-202.

21. Damasceno VO, Vianna VRA, Vlianna JM, Lacio M, Lima JRP, Novaes JS. Imagem corporal e corpo ideal. Rev Bras Cien Mov 2006; 14(1):87-96.

22. Hausenblas HA, Fallon EA. Exercise and body image: A meta-analysis. Psychol Health 2006; 21(1):33-47.

23. Ciampo LA Del, Rodrigues DMS, Ciampo IRL Del, Cardoso VC, Bettiol H, Barbieri MA. Percepção corporal e atividade física em uma coorte de adultos jovens brasileiros. J Hum Growth Dev 2010; 20(3):671.

24. Stein C, Fisher L, Berkey C, Colditz G. Adolescent Physical Activity and Perceived Competence: Does Change in Activity Level Impact Self-Perception? J Adolesc Heal 2007; 40(5):462.e1-462.e8.

25. Finne E, Bucksch J, Lampert T, Kolip P. Age, puberty, body dissatisfaction, and physical activity decline in adolescents. Results of the German Health Interview and Examination Survey (KiGGS). Int J Behav Nutr Phys Act 2011; 8(1):119.

26. Abbott BD, Barber BL. Differences in functional and aesthetic body image between sedentary girls and girls involved in sports and physical activity: Does sport type make a difference? Psychol Sport Exerc 2011; 12(3):333-342.

27. Inttituto Nacional de Estudos e Pesquisas Educacionais Anísio Teixeira (INEP). Censo Escolar de Educação Básica 2013. Brasília: INEP; 2013.

28. De Onis M, Onyango AW, Borghi E, Siyam A, Nishida C, Siekmann J. Development of a WHO growth reference for school-aged children and adolescents. Bull World Health Organ 2007; 85(10):812-819.

29. Farias Júnior JC, Lopes AS, Mota J, Santos MP, Ribeiro JC, Hallal PC. Validade e reprodutibilidade de um questionário para medida de atividade física em adolescentes: uma adaptação do Self-Administered Physical Activity Checklist. Rev Bras Epidemiol 2012; 15(1):198-210.

30. Tanner JM. Growth at adolescence. $2^{\mathrm{a}}$ ed. Oxford: Blackwell; 1962.

31. Bojikian LP, Massa M, Martin RHC, Teixeira CP, Kiss MAPD, Bohme MTS. Auto-avaliação puberal feminina por meio de desenhos e fotos. Rev Bras Atividade Física Saúde 2002; 7(2):24-34.

32. Martin RHC, Uezu R, Parra AS, Arena SS, Bojikian LP, Bohme MTS. Auto-avaliação da maturação sexual masculina por meio da utilização de desenhos e fotos. Rev Paul Educ Física 2001; 15(2):212-222.

33. Associação Brasileira de Empresas e Pesquisa (ABEP). Critério de Classificação Econômica Brasil 2013 [Internet]. 2012 [acessado 2018 Maio 14]. Disponível em: http://www.abep.org/new/criterioBrasil.aspx 
34. Cooper PJ, Taylor MJ, Cooper Z, Fairbum CG. The development and validation of the body shape questionnaire. Int J Eat Disord 1987; 6(4):485-494.

35. Conti MA, Cordás TA, Latorre MRDO. A study of the validity and reliability of the Brazilian version of the Body Shape Questionnaire (BSQ) among adolescents. Rev Bras Saúde Matern Infant 2009; 9(3):331-338.

36. Thompson MA, Gray JJ. Development and Validation of a New Body-Image Assessment Scale. J Pers Assess 1995; 64(2):258-269.

37. Gardner RM, Brown DL. Body image assessment: A review of figural drawing scales. Pers Individ Dif 2010; 48(2):107-111.

38. Paludo J, Dalpube V. Imagem corporal e sua relação com o estado nutricional e a qualidade de vida de adolescentes de um município do interior do Rio Grande do Sul. Nutrire 2015; 40(1):1-9.

39. Daniel CG, Guimarães G, Aerts DRGC, Rieth S, Batista RRA, Jacob MH. Imagem corporal, atividade física e estado nutricional em adolescentes no sul do Brasil. Aletheia 2014; 45:128-138.

40. Instituto Brasileiro de Geografia e Estatística (IBGE). Pesquisa Nacional de Saúde do Escolar: 2015. Rio de Janeiro: IBGE; 2016.

41. Fidelix YL, Silva DAS, Pelegrini A, Silva AF, Petroski EL. Insatisfação com a imagem corporal em adolescentes de uma cidade de pequeno porte: associação com sexo, idade e zona de domicílio. Rev Bras Cineantropometria Desempenho Hum 2011; 13(3):202-207.

42. Vilela JEM, Lamounier JA, Dellaretti Filho MA, Barros Neto JR, Horta GM. Eating disorders in school children. J Pediatr (Rio J) 2004; 80(1):49-54.

43. Adami F, Frainer DES, Santos JS, Charles T, De-Oliveira FR. Insatisfação Corporal e Atividade Física em Adolescentes da Região Continental. Psicol Teor Pesqui 2008; 24(2):143-149.
44. Mota DCL, Laus MF, Almeida SS, Costa TMB, Moreira RCM. Imagem Corporal e suas Relações com a Atividade Física. Psico 2012; 43(2):237-242.

45. Morgan CM, Ramalho I, Negrão B. Etiologia dos transtornos alimentares: aspectos biológicos, psicológicos e sócio - culturais. Rev Bras Psiquiatr 2002; 24(Supl. III):18-23.

46. Alves D, Pinto M, Alves S, Mota A, Leirós V. Cultura e imagem corporal. Motricidade 2009; 5(1):1-20.

47. Silva ACS, Sales ZN, Moreira RM, Boery EN, Santos WS, Teixeira JRB. Representações socisid de adolescentes sobre ser saudável. Rev Bras Cien Esporte 2014; 36(2):397-409.

48. Sampaio RPA, Ferreira RF. Beleza, identidade e mercado. Psicol em Rev 2009; 15(1):120-140.

49. Pimentel ZNS, Aerts DRGC, Jacob MHVM, Alves GG, Câmara SG, Palazzo L. Preocupação com a imagem corporal e fatores associados em adolescentes do ensino público em um município da Amazônia. Adolescência Saúde 2017; 14(2):94-103.

Artigo apresentado em 25/05/2018

Aprovado em 11/02/2019

Versão final apresentada em 13/02/2019 